\title{
A functional differentiation of STM and LTM
}

KEITH G. SCOTT, ARTHUR E. WHIMBEY AND CASEY DUNNING

UNIVERSITY OF ILLINOIS

The study investigated the recovery from within list proactive inhibition while recalling a previously learned item. It is shown that while performing on this item in LTM recovery from proactive inhibition in STM occurred. The result indicates that STM and LTM refer to more than just a time dimension, thus confirming previous findings.

In a previous paper (Scott et al, 1967) it was shown that not only was Proactive Inhibition (PI) in ShortTerm Memory (STM) not generated by performing on a previously learned trigram, but that it appeared to dissipate under this condition. This paper aims to provide a clearer demonstration of this effect.

The importance of the observation stems from the disputed distinction between STM and LTM (Melton, 1963). Typical arguments for a single continuum have been based on the observed similarity of the laws governing STM and LTM. Differences in the mode of operation of STM and LTM have recently been reported by Bradley \& Dale (1966) as well as by the present authors. This study examines the question further by investigating the interaction between STM, LTM, and growth of within list PI.

\section{Method}

The Ss were 32 males from a subject pool of undergraduate students. None of the Ss had previously served in a verbal learning study. Each $S$ was randomly assigned to a stimulus list on appearance.

The stimuli were 16 trigrams chosen from the $25 \%$ association value list of Witmer (Underwood \& Schultz, 1960). They were chosen so that first, no consonant was used more than three times over the 16 trigrams, and second, no consonant appeared three times in the same intra-trigram serial position. From these 16 trigrams, 32 random order lists were made with t'ie restriction that a particular trigram was used for exactly two Ss as the pretrained or "old" trigram.

Other materials were three digit numbers, greater than 175, taken from a table of random numbers. These were presented to control rehearsal after the training presentation of a syllable. The $S$ was instructed to count backwards from this number by threes, pacing himself with a small light that flickered once per sec.

The general procedure of Peterson \& Peterson (1959) was followed. The materials were photographed and projected onto a wall by a slide projector controlled by a tape programmer. The timing employed was as follows: the word recidy appeared for 4 sec., the trigram was shown for $2 \mathrm{sec}$., the number appeared and was displayed during the counting interval of 27 sec., and finally, three dots appeared for $8 \mathrm{sec}$. to signal for the S's recall.
After simple instructions the Ss began a session with a pretraining block of five presentations and recalls of one of the items. This was followed by a 2 min. rest. Next came the experimental session proper with the learning and recall of 25 trigrams presented without any rest.

These trigrams consisted of alternate blocks of two kinds. In the one kind all the presentations were of five trigrams randomly assigned from the list. In the other kind the block consisted of five presentations of the same trigram that had been pretrained at the start of a session. These blocks are called New and old, respectively. The order of presentation was, pretraining, a rest of 2 min., New, Old, New, Old, New. Results and Discussion

Figure 1 shows the generation of PI within the blocks of new items, and the dissipation of PI during the blocks of five trials on a previously learned item. The McNemar test for correlated proportions showed that for blocks 1, 3, and 5 performance on item 5 was significantly poorer than on item 1 , indicating that PI was in fact generated during the block of new items $(z=2.18, p<.02 ; z=2.18, p<.02 ; z=3.75, p<.001$ for blocks 1,3 , and 5 , respectively). To determine whether the PI completely dissipated during the block of five trials with the old trigram, item 1 of blocks 3 and 5 item 1 of block 3 was not significantly poorer than on item 1 of block $1(z=.578, p>.28)$ and there were actually fewer errors made on item 1 of block 5 than item 1 of block 1. It therefore appears that five trials on a previously learned item allowed PI in STM to completely dissipate.

In an earlier paper (Scott et al, 1967) it was reported that a trial consisting of a previously learned trigram did not generate PI in STM. This was interpreted as evidence for a dual theory of memory, an LTM and an STM system. The results of this experiment present

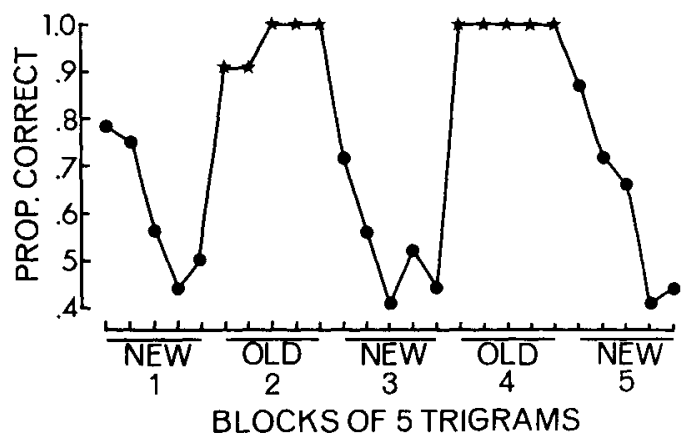

Fig. 1. The proportion of correct recalls as a function of the ordinal position in a block of old or new trigrams. was compared with item 1 of block 1. Performance on 
even stronger evidence for two separate systems. Using the terms LTM and STM in a purely descriptive sense to refer to the time dimension, as was done by Melton (1963), it has been shown that one type of activity can occur in LTM (the recall of a previously learned item) while a different activity is occurring in STM (the dissipation of PI). This appears to indicate that LTM and STM refer to more than just the time dimension, but rather refer to two functional systems.

The distinction between LTM and STM in the present experiment consists of describing previous and well learned items as in LTM and individual singly presented items as in STM. These could represent extreme values of a single independent variable, degree of learning, as shown by its effect on within-session performance. The results would thus be compatible with a single mechanism theory. A critical test of the question requires that a detailed model of memory be presented by the advocates of each position. For example, a dual-mechanism model might postulate than an item move from STM to LTM in a discontinuous jump. Such a theory would be supported by an experiment showing a performance discontinuity as items pass from STM to LTM. The major problem of such a design is that discontinuous data are particularly susceptible to distortion due to averaging (Estes, 1956) and a negative, but artifactual outcome is likely. However a dual-mechanism model does not intrinsically require a discontinuous process; an item might move gradually from STM to LTM. A dual-mechanism theory of this latter type would in many cases make exactly the same predictions as a single-mechanism theory and as such it would be harder to devise an experiment which would distinguish between the positions.

In any case, the functional characteristics of items which have been well learned on previous trials will have to be taken into account by any theory of memory. This account may include degree of previous learning, the formation of a "structure" (Mandler, 1962) by well learned associations preventing them from interfering, or some other memory mechanism. For the present it appears that a dual-mechanism theory will be able to account for these findings more easily and consistently than a single-mechanism theory.

\section{References}

Bradley, A. D., \& Dale, H. C. The effect of semantic similarity on retroactive interference in long- and short-term memory. $J$. verbal Learn. verbal Behav., 1966, 5, 417-420.

Estes, W. $\mathbf{K}$. The problem of inference from curves based on group data. Psychol. Bull., 1956, 53, 134-140.

Mandler, George A. From association to structure. Psychol. Rev., $1962,5,415-427$.

Melton, A. W. Implications of short-term memory for a general theory of memory. J. verbal Learn. verbal Behav., 1963, 2, 1-21.

Peterson, L. R., \& Peterson, M. J. Short-term retention of individual verbal items. J. exp. Psychol., 1959, 58, 193-198.

Scott, K. G., Whimbey, A. E., \& Dunning, C. Separate LTM and STM systems? Psychon. Sci., 1967, 7, 55-56.

Underwood, B. L., \& Shultz, R. W. Meaningfulness and verbal learning. Chicago: Lippincott, 1960.

\section{Nore}

1. This research was supported in part by Grant MH07346 from the National Institutes of Health and in part by an NSF Undergraduate summer research program No. NSF 6Y 113 in which the junior author was a participant. 